\title{
Efficient synthesis and antimicrobial evaluation of some Mannich bases from 2-arylidine-1-thia-4- azaspiro[4.5]decan-3-ones
}

Essam M Hussein ${ }^{1,2^{*}}$, Ghada S Masaret ${ }^{1}$ and Khalid S Khairou ${ }^{1}$

\begin{abstract}
Background: Thiazolidinone, has been employed in the preparation of different important drugs required for treatment of inflammations, bacterial infections, and hypertension. Mannich bases have been shown to exhibit diverse biological activities, such as antibacterial, and antifungal activities. Spiroheterocycles including thiazolidine moiety have antimicrobial activity.

Results: In this study, a novel, rapid, and efficient protocol is developed for the synthesis of various 2-arylidine-1-thia-4azaspiro[4.5]decan-3-ones using sodium dodecylbenzene sulfonate (DBSNa) as an inexpensive and readily available reagent in acetic acid at room temperature. High yields, easy work-up, and short reaction times are advantages of this procedure. The synthesized arylidines were undergone Mannich reaction with formaldehyde and secondary amines in absolute ethanol at room temperature to afford the corresponding N-Mannich bases. All prepared Mannich bases were evaluated for their antimicrobial activity.
\end{abstract}

Conclusions: Good activity was noted for Mannich bases from 2-arylidine-1-thia-4-azaspiro[4.5]decan-3-ones, with some members recorded higher antimicrobial activity.

Keywords: Spiro, 1-thia-4-azaspiro[4.5]decan-3-one, Sodium dodecylbenzene sulfonate, Antimicrobial activity, Mannich bases

\section{Background}

There are many bioactive molecules which possess various heteroatoms such as nitrogen, sulfur and oxygen, always taken the attention of chemists over the years mainly because of their biological significance. Thiazolidinones are thiazolidine derivatives which have a sulfur atom at position 1, a nitrogen atom at position 3 and a carbonyl group at position 2, 4, or 5 [1], is considered as an important biologically active scaffold that possesses almost all types of biological activities [2]. This heterocyclic system has been employed in the preparation of different important drugs required for treatment of inflammations [3], bacterial infections [4], and hypertension [5]. Some of the thiazole analogues are used as

\footnotetext{
*Correspondence: essam.hussein78@yahoo.com

${ }^{1}$ Department of Chemistry, Faculty of Applied Sciences, Umm Al-Qura University, Makkah, Saudi Arabia

${ }^{2}$ Department of Chemistry, Faculty of Science, Assiut University, Assiut 71516, Egypt
}

fungicides, inhibiting in vivo the growth of xanthomonas and as ingredients of herbicides, antischistosomicidal, and anthelmintic drugs [6]. Mannich bases are reported to show a diversity of biological activities, such as antibacterial $[7,8]$, antifungal $[9,10]$ activities. Spiro derivatives have antibacterial, anticancer, and anticonvulsants activities. Spiro heterocycles were used as nitric oxide synthesis inhibitors [11] and potential topical agents for vaginal infection [12]. Spiro heterocyclic compounds including thiazolidine moiety have antimicrobial activity [13].

In this paper and as a consequence of our previous work on the synthesis of $N$-heterocyclic compounds [14-18], and bioactive heterocyclic agents [19-21], we reported herein an efficient protocol to the synthesis of $\mathrm{N}$-Mannich bases (6a-r) from 2-arylidine-1-thia-4-azaspiro[4.5]decan-3-ones (5a-f). The anti-microbial activity of the prepared compounds (6a-r) was screened. 


\section{Results and discussion Chemistry}

First of all, 1-thia-4-azaspiro[4.5]decan-3-one (3) was prepared via the three component cyclocondensation reaction of cyclohexanone, thioglycolic acid, and ammonium carbonate according to the previously reported procedure [22] as shown in Scheme 1.

\section{Synthesis of 2-arylidine-1-thia-4-azaspiro[4.5]decan-3-ones (5a-f)}

In the first part of our research, we investigated a novel, rapid and efficient protocol that was developed for the synthesis of some 2-arylidine-1-thia-4-azaspiro[4.5]decan3-ones $(\mathbf{5 a}-\mathbf{f})$ by the condensation of 1-thia-4-azaspir$\mathrm{o}$ [4.5]decan-3-one (3) with aromatic aldehydes (4a-f) using sodium dodecylbenzene sulfonate (DBSNa) (20 mol \%) in acetic acid at room temperature as shown in Scheme 2 and Table 1.

To find out the suitable conditions for the synthesis of 2-arylidine-1-thia-4-azaspiro[4.5]decan-3-ones, a series of experiments were performed with the standard reaction of 1-thia-4-azaspiro[4.5]decan-3-one (3) and benzaldehyde (4a) as a model reaction (Scheme 3, Table 2).

Effect of the reaction conditions In our initial study, we tried to optimize the model procedure mentioned above by detecting the efficiency of different reaction conditions, such as piperidine/EtOH, $p$-TSA/EtOH, AcOH/ $\mathrm{EtOH}, \mathrm{AcONa} / \mathrm{AcOH}, \mathrm{H}_{2} \mathrm{SO}_{4} / \mathrm{AcOH}, \mathrm{DBSNa} / \mathrm{MeOH}$, DBSNa/EtOH, DBSNa/ $\mathrm{H}_{2} \mathrm{O}, \mathrm{DBSNa} / \mathrm{AcOH}$ (Table 2).

In each case, the reactants $(10 \mathrm{mmol})$ were allowed together in $10 \mathrm{~mL}$ solvent at room temperature. In the case of piperidine/EtOH, p-TSA/EtOH, AcOH/EtOH, and $\mathrm{AcONa} / \mathrm{AcOH}$, the reaction proceeded with comparatively longer reaction time and poor reaction yield (Table 2, entries 1-4). Acetic acid acidified with a drop of $\mathrm{H}_{2} \mathrm{SO}_{4}$ can push the reaction towards the formation of product in yields of $61 \%$ (Table 2, entry 5).

In the presence of sodium dodecylbenzene sulfonate (DBSNa), the reaction was possible and the product (5a) was obtained in good yields.

Sodium dodecylbenzene sulfonate was used in different reaction media such as ethanol, methanol, water, and acetic acid (Table 2, entries 6-9). The best results were obtained when DBSNa was used as catalyst in acetic acid as reaction medium, which provided a yield of $94 \%$ (Table 2, entry 8).

Unfortunately, when the reaction was performed in water, the yield of the desired product was obtained in a trace amount (Table 2, entry 9).

Evaluation of catalytic activity of DBSNa To determine the appropriate concentration of the catalyst used, we investigated the model reaction at different concentrations of $\operatorname{DBSNa}(5,10,15,20$, and $25 \mathrm{~mol} \%)$. It was found that when the amount of DBSNa was increased from 5 to $20 \mathrm{~mol} \%$, the yield increased from 68 to $94 \%$, respectively. However, there was no significant change in reaction yield when the amount of catalyst was increased further, to $25 \mathrm{~mol} \%$. Thus, $20 \mathrm{~mol} \% \mathrm{DBSNa}$ in acetic acid is sufficient to push this reaction forward (Table 3).

\section{Synthesis of Mannich bases of 2-arylidine-1-thia-4- azaspiro[4.5]decan-3-ones (6a-r)}

The second part of the research includes the preparation of a series of Mannich bases (6a-r) in good yield (71-91\%) by the reaction of 2-arylidine-1-thia-4-azaspiro[4.5]decan-3-ones $(\mathbf{5 a}-\mathbf{f})$ with formaldehyde and secondary amines (piperidine, morpholine, and pyrrolidine) in absolute ethanol at room temperature for 1.5$4 \mathrm{~h}$ (Scheme 4 and Table 4).

The structures of the isolated new products $(6 \mathbf{6}-\mathbf{r})$ were deduced by analyzing their physical and spectroscopic data, such as the data obtained using IR, ${ }^{1} \mathrm{H}$ NMR, and ${ }^{13} \mathrm{C}$ NMR spectroscopy.

Taking (6b) as an example, the IR spectrum showed the lack of the absorption band corresponding to $\mathrm{NH}$ group. The ${ }^{1} \mathrm{H}$ NMR spectrum showed the presence of a singlet signal at $\delta=4.15 \mathrm{ppm}$ for the methylene protons and two triplet signals at 2.47, 3.59, ppm for morpholine ring protons.

\section{Antimicrobial activity \\ In vitro antibacterial activity}

The synthesized compounds (6a-r) were screened in vitro for their antibacterial at $50 \mathrm{mg} / \mathrm{mL}$ concentration against Staphylococcus aureus as Gram positive bacteria, Escherichia Coli, Pseudomonas aeroginosa as Gram negative bacteria using Ciprofloxacin as standard antibacterial reference.

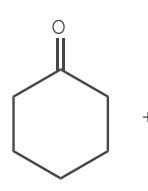

(1)

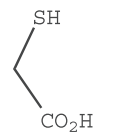

(2)
$\left(\mathrm{NH}_{4}\right)_{2} \mathrm{CO}_{3}$

$\underset{\text { Dean-Stark }}{\stackrel{\text { Dry Toluene }}{\longrightarrow}}$

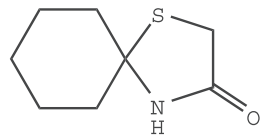

(3)

Scheme 1 Synthesis of 1-thia-4-azaspiro[4.5]decan-3-one (3). 


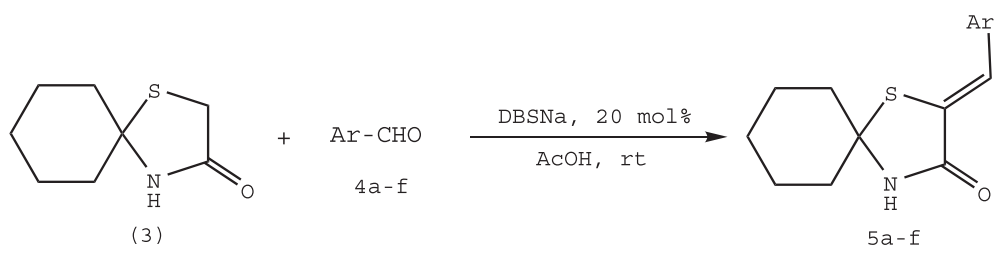

Scheme 2 Synthesis of 2-arylidine-1-thia-4-azaspiro[4.5]decan-3-ones (5a-f).

Most of the tested compounds showed excellent antibacterial activities with respect to the reference drug.

The results obtained in Table 5 indicated that the type of substituents $(A r)$ and $(R)$ are the controlling factors in developing the total antibacterial properties of the tested Mannich bases (6a-r).

Data in Table 5 revealed that compounds $6 \mathbf{e}, \mathbf{6 ~ h}, \mathbf{6} \mathbf{k}$, $\mathbf{6} \mathbf{m}, \mathbf{6 n}, \mathbf{6 p}$ and $\mathbf{6 q}$, have superior significant antibacterial potency. Compounds $\mathbf{6 n}$ and $\mathbf{6 q}\left[\mathrm{Ar}=4-\mathrm{MeOC}_{6} \mathrm{H}_{4}\right.$ and 4pyridyl; $R=$ morpholin-1-yl] have excellent activities against Gram positive bacteria (Staphylococcus aureus) and Gramnegative bacteria (Escherichia Coli and Pseudomonas aeroginosa). Compound 6p [ $A r=4$-pyridyl; $R=$ piperidin-1-yl] has excellent potency against Staphylococcus aureus, moderate activity against Pseudomonas aeroginosa, and poor activity against Escherichia Coli. Structure-Activity relationships (SAR) based on the obtained results indicated that the best observed antibacterial activity is that which $A r$ is phenyl ring attached with electron donating function $(\mathrm{MeO})$ as exhibit in compound $\mathbf{6 n}$. However, substituting the phenyl ring with electron withdrawing function $(\mathrm{Cl}$, $\mathrm{NO}_{2}$ ) the antibacterial behavior is decreased. When $\mathrm{Ar}$ is unsubstituted phenyl ring the antibacterial activity is not significant. It has also, been noticed that when $A r$ is pyridine ring exhibited in (6p) and (6q) increase in the observed antibacterial properties was noticed compared with the case of using phenyl ring.

\section{In vitro antifungal activity}

With respect to antifungal activity, the synthesized compounds were screened against three fungal strains;

Table 1 Synthesis of the 2-arylidine-1-thia-4azaspiro[4.5]decan-3-ones (5a-f) using DBSNa (15 mol\%)

\begin{tabular}{lllll}
\hline Entry & Product $^{\mathbf{a}}$ & $\mathbf{A r}$ & Time (min) & Yield $^{\mathbf{b}}(\%)$ \\
\hline 1 & $\mathbf{5 a}$ & $\mathrm{Ph}$ & 60 & 94 \\
2 & $\mathbf{5 b}$ & $4-\mathrm{ClC}_{6} \mathrm{H}_{4}$ & 50 & 96 \\
3 & $\mathbf{5 c}$ & $4-\mathrm{BrC}_{6} \mathrm{H}_{4}$ & 60 & 93 \\
4 & $\mathbf{5 d}$ & $4-\mathrm{O}_{2} \mathrm{NC}_{6} \mathrm{H}_{4}$ & 45 & 87 \\
5 & $\mathbf{5 e}$ & $4-\mathrm{MeOC}_{6} \mathrm{H}_{4}$ & 40 & 98 \\
6 & $\mathbf{5 f}$ & $4-p y r i d y l$ & 70 & 90 \\
\hline
\end{tabular}

${ }^{a}$ Reaction conditions: 1-thia-4-azaspiro[4.5]decan-3-one (3) (10 mmol), aromatic aldehydes (4a-f) $(10 \mathrm{mmol})$, and DBSNa (20 mol\%) in $10 \mathrm{~mL}$ acetic acid at room temperature.

b Isolated yields.
Aspergillus niger, Candida albicans, Fusarium oxysporium using Nystatin as standard antifungal reference (at $50 \mathrm{mg} / \mathrm{mL}$ concentration). Most of the tested compounds showed excellent antibacterial activities with respect to the reference drug.

As antibacterial activity, the obtained results indicated that the type of substituents $(A r)$ and $(R)$ are the controlling factors in developing the antifungal properties of the tested compounds (6a-r). Results of antifungal activities were shown in Table 6 . Data in Table 6 showed that compounds $\mathbf{6 d}, \mathbf{6} \mathbf{l}$, and $\mathbf{6 p}$ have remarkable antifungal potency. Compounds $6 \mathbf{l}\left[A r=4-\mathrm{O}_{2} \mathrm{NC}_{6} \mathrm{H}_{4} ; R=\right.$ piperidin-1-yl] exhibit excellent activities against Candida albicans and Fusarium oxysporium as well as good potency against Aspergillus niger. Compound 6p $[\mathrm{Ar}=4$ pyridyl; $R=$ piperidin-1-yl] has excellent activity against Aspergillus niger as well as good potency against Candida albicans and Fusarium oxysporium. Compound $\mathbf{6 d}$ $\left[\mathrm{Ar}=4-\mathrm{ClC}_{6} \mathrm{H}_{4} ; R=\right.$ piperidin-1-yl] exhibit good potency against Aspergillus niger, Candida albicans and Fusarium oxysporium. Structure-Activity relationships (SAR) based on the obtained results indicated that the best observed antifungal activity is that which $A r$ is phenyl ring attached with electron withdrawing function $\left(\mathrm{NO}_{2}, \mathrm{Cl}\right)$ as exhibit in compound $\mathbf{6} \mathbf{1}$ and $\mathbf{6 d}$, respectively. However, substituting the phenyl ring with electron donating function $(\mathrm{MeO})$ the antifungal behavior is decreased. When $A r$ is unsubstituted phenyl ring the antifungal activity is not remarkable. It has also, been noticed that when $A r$ is pyridine ring exhibited in (6p) increase in the observed potent antifungal properties was noticed compared with the case of using phenyl ring.

\section{Conclusions}

The authors have developed a novel, rapid and efficient protocol for the synthesis of various 2-arylidine-1-thia-4azaspiro[4.5]decan-3-ones using sodium dodecylbenzene sulfonate (DBSNa) in acetic acid at room temperature. The results clearly demonstrate that the using of the sodium dodecylbenzene sulfonate as an inexpensive and readily available reagent markedly enhances the efficiency of the chemical processes of interest. Mannich bases from the synthesized 2-arylidine-1-thia-4-azaspiro[4.5]decan-3ones were achieved and evaluated as antimicrobial agents and showed remarkable activities. 


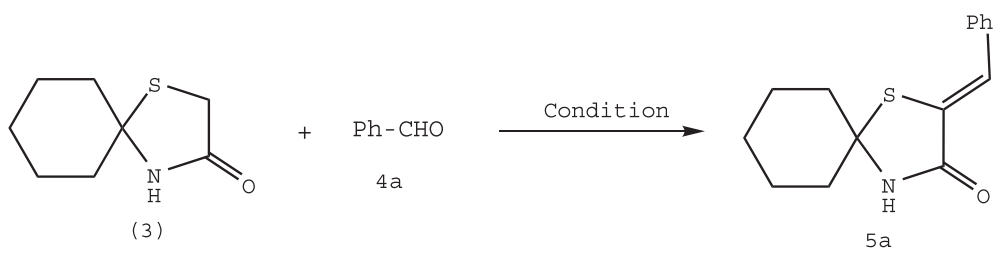

Scheme 3 Synthesis of 2-benzylidine-1-thia-4-azaspiro[4.5]decan-3-ones (5a).

\section{Experimental \\ Chemistry}

General methods The time required for completion of each reaction was monitored by TLC. All melting points are uncorrected and were measured on a Gallenkamp apparatus. The IR spectra were recorded on a Shimadzu $470 \mathrm{IR}$ spectrometer $(\mathrm{KBr})$ vmax, $\mathrm{cm}^{-1}$. The ${ }^{1} \mathrm{H}$ and ${ }^{13} \mathrm{C}$ NMR spectra were measured on a Varian EM-200 $(1 \mathrm{H}$ : $400 \mathrm{MHz},{ }^{13} \mathrm{C}: 100 \mathrm{MHz}$ ) spectrometer with TMS as internal standard. Mass spectra were determined on a JEOL JMS-600 spectrometer. Elemental analyses $(\mathrm{C}, \mathrm{H}$, $\mathrm{N}$, and $\mathrm{S}$ ) were performed on an elemental analysis system $\mathrm{GmbH}$ VarioEL $\mathrm{V}_{2.3}$.

\section{General procedure for synthesis of 2-Arylidine-1-thia-4- azaspiro[4.5]decan-3-ones (5a-f)}

To a solution of (3) $(1.71 \mathrm{~g}, 10 \mathrm{mmol})$ in acetic acid $(10 \mathrm{~mL})$, corresponding aromatic aldehyde $(10 \mathrm{mmol})$ was added. Then DBSNa (20 mol\%) was added and the reaction mixture was stirred at room temperature for the desired time as monitored by TLC (Table 1). After completion of the reaction, the solid product was filtered and washed with cold water, dried, and recrystallized from ethanol (95\%).

Table 2 The effect of reaction condition on the synthesis of (5a) under various conditions ${ }^{a}$

\begin{tabular}{lllll}
\hline Entry & Solvent $^{\mathbf{b}}$ & Catalyst $^{\mathbf{c}}$ & Time (min) & Yield $^{\mathbf{d}} \mathbf{( \% )}$ \\
\hline 1 & $\mathrm{EtOH}$ & Piperidine & 180 & 37 \\
2 & $\mathrm{EtOH}$ & $\mathrm{AcOH}$ & 180 & 43 \\
3 & $\mathrm{EtOH}$ & $p^{-T S A}$ & 180 & 40 \\
4 & $\mathrm{AcOH}$ & $\mathrm{AcONa}$ & 150 & 46 \\
5 & $\mathrm{AcOH}$ & $\mathrm{H}_{2} \mathrm{SO}_{4}$ & 120 & 61 \\
6 & $\mathrm{EtOH}$ & $\mathrm{DBSNa}$ & 120 & 66 \\
7 & $\mathrm{MeOH}$ & $\mathrm{DBSNa}$ & 120 & 64 \\
8 & $\mathrm{AcOH}$ & $\mathrm{DBSNa}$ & 60 & 94 \\
9 & $\mathrm{H}_{2} \mathrm{O}$ & DBSNa & 180 & trace \\
\hline
\end{tabular}

${ }^{a}$ The reaction was carried out with 1-thia-4-azaspiro[4.5]decan-3-one (3)

$(10 \mathrm{mmol})$, benzaldehydes $(4 \mathrm{a})(10 \mathrm{mmol})$ at room temperature.

b $10 \mathrm{~mL}$ solvent.

c $20 \mathrm{~mol} \%$.

d Isolated yields.

\section{2-Benzylidene-1-thia-4-azaspiro[4.5]decan-3-one (5a)}

Pale yellow crystals; mp 200-202 ${ }^{\circ} \mathrm{C}$; IR: $3200(\mathrm{NH}), 3020$ (CH arom.), 1700 (C=O), 695 (C-S); ${ }^{1} \mathrm{H}$ NMR (DMSO$\left.\mathrm{d}_{6}\right)$ : 1.10-1.95 (m, 10H, $\left.5 \times \mathrm{CH}_{2}\right), 7.50(\mathrm{~m}, 5 \mathrm{H}, \mathrm{Ph}-\mathrm{H})$, 7.85 (s, 1H, CH), 8.97 (s, 1H, NH, D $2 \mathrm{O}$-exchangeable) ppm; ${ }^{13} \mathrm{C}$ NMR: $21.7\left(2 \mathrm{CH}_{2}\right), 24.8\left(\mathrm{CH}_{2}\right), 25.6\left(2 \mathrm{CH}_{2}\right)$, 47.9 (spiro C), $122.3(\mathrm{CH}), 124.8(2 \mathrm{CH}), 126.1(2 \mathrm{CH})$, 129.4 (C), $131.1(\mathrm{C}), 136.7$ (C), $170.2(\mathrm{C}=\mathrm{O})$ ppm; EIMS, $m / z$ (\%): 260.10 (8) $[\mathrm{M}+1], 259.01$ (54) $[M+]$, 216.05 (100.0). Calc. for $\mathrm{C}_{15} \mathrm{H}_{17} \mathrm{NOS}$ : C, 69.46; H, 6.61; $\mathrm{N}, 5.40 ; \mathrm{S}, 12.36$. Found: C, 69.28; H, 6.47; N, 5.24; S, 12.16 .

\section{2-(4-Chlorobenzylidene)-1-thia-4-azaspiro[4.5]decan-3-one} (5b)

Pale yellow crystals; mp 210-212 ${ }^{\circ}$; IR: 3190 (NH), 3085 (CH arom.), 1695 (C= O), 695 (C-S); ${ }^{1} \mathrm{H}$ NMR (DMSO$\left.\mathrm{d}_{6}\right)$ : 1.15-2.10 (m, 10H, $\left.5 \times \mathrm{CH}_{2}\right), 7.35(\mathrm{~d}, 2 \mathrm{H}, 2 \mathrm{CH}, J=$ 7.4), 8.00 (d, 2H, 2CH, J=7.4), $8.90(\mathrm{~s}, 2 \mathrm{H}, \mathrm{CH}+\mathrm{NH})$ ppm; EI-MS, $m / z$ (\%): 293.54 (44) $[M+], 258.15$ (30), 250.02 (100.0). Calc. for $\mathrm{C}_{15} \mathrm{H}_{16}$ ClNOS: C, 61.32; $\mathrm{H}$, 5.49; Cl, 12.07; N, 4.77; S, 10.91. Found: C, 61.12; H, 5.30; Cl, 11.88; N, 4.59; S, 10.78 .

\section{2-(4-Bromobenzylidene)-1-thia-4-azaspiro[4.5]decan-3-one} (5c))

Pale yellow crystals; mp 221-222 ${ }^{\circ} \mathrm{C}$; IR: $3200(\mathrm{NH}), 3090$ (CH arom.), 1695 (C = O), 695 (C-S); ${ }^{1} \mathrm{H}$ NMR (DMSO$\left.\mathrm{d}_{6}\right)$ : 1.00-2.10 (m, 10H, $\left.5 \times \mathrm{CH}_{2}\right), 7.40(\mathrm{~d}, 2 \mathrm{H}, 2 \mathrm{CH}, J=$ 7.2), 8.10 (d, 2H, 2CH, J=7.2), 8.60 (s, 1H, CH), 10.10 (s, $1 \mathrm{H}, \mathrm{NH}) \mathrm{ppm}$; EI-MS, $\mathrm{m} / z(\%): 339.01$ (20) $[M+]$, 296.15 (100.0), 79 (15). Calc. for $\mathrm{C}_{15} \mathrm{H}_{16}$ BrNOS: C,

Table 3 Evaluation of catalytic activity of DBSNa in the synthesis of $(5 a)^{a}$

\begin{tabular}{llll}
\hline Entry & Amount of DBSNa (mol \%) & Time $(\boldsymbol{m i n})$ & Yield $^{\mathbf{b}} \mathbf{( \% )}$ \\
\hline 1 & 5 & 90 & 68 \\
2 & 10 & 70 & 81 \\
3 & 15 & 60 & 87 \\
4 & 20 & 60 & 94 \\
5 & 25 & 60 & 94
\end{tabular}

${ }^{a}$ The reaction was carried out with 1-thia-4-azaspiro[4.5]decan-3-one (3) $(10 \mathrm{mmol})$, benzaldehydes $(\mathbf{4 a})(10 \mathrm{mmol})$ and DBSNa in $10 \mathrm{~mL}$ acetic acid at room temperature.

${ }^{\mathrm{b}}$ Isolated yields. 


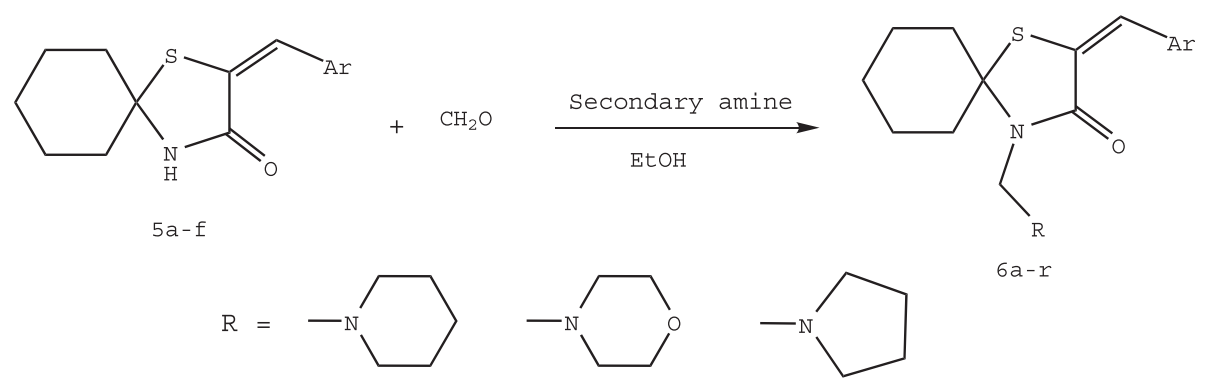

Scheme 4 Synthesis of Mannich bases (6a-r).

53.26; H, 4.77; Br, 23.62; N, 4.14; S, 9.48. Found: C, 53.00; H, 4.80; Br, 23.47; N, 3.81; S, 9.19.

\section{2-(4-Nitrobenzylidene)-1-thia-4-azaspiro[4.5]decan-3-one}

\section{$(5 d))$}

Yellow crystals; mp 249-250 C; IR: $3220(\mathrm{NH}), 3090(\mathrm{CH}$ arom.), 1690 (C=O), 690 (C-S); ${ }^{1} \mathrm{H}$ NMR (DMSO-d 6 ): 1.20-2.10 (m, 10H, $\left.5 \times \mathrm{CH}_{2}\right), 8.10(\mathrm{~d}, 2 \mathrm{H}, 2 \mathrm{CH}, J=7.4)$, 8.50 (d, 2H, 2CH, J = 7.4), 8.70 (s, $1 \mathrm{H}, \mathrm{CH}), 10.20$ (s, 1H, $\mathrm{NH}$ ) ppm; EI-MS, $m / z$ (\%): 304.61 (29) [M+], 261.25 (100.0). Calc. for $\mathrm{C}_{15} \mathrm{H}_{16} \mathrm{~N}_{2} \mathrm{O}_{3} \mathrm{~S}: \mathrm{C}, 59.19 ; \mathrm{H}, 5.30 ; \mathrm{N}, 9.20$; S, 10.54. Found: C, 58.89; H, 5.43; N, 9.00; S, 10.30 .

Table 4 Synthesis of the Mannich bases (6a-r) ${ }^{a}$

\begin{tabular}{|c|c|c|c|c|}
\hline Mannich product & $\mathrm{Ar}$ & $\mathbf{R}$ & Time (h) & Yield $^{\mathrm{b}}(\%)$ \\
\hline $6 a$ & $\mathrm{Ph}$ & piperidin-1-yl & 2.0 & 80 \\
\hline $6 b$ & $\mathrm{Ph}$ & morphilin-1-yl & 3.0 & 76 \\
\hline $6 c$ & $\mathrm{Ph}$ & pyrrolidin-1-yl & 2.5 & 79 \\
\hline $6 d$ & $4-\mathrm{ClC}_{6} \mathrm{H}_{4}$ & piperidin-1-yl & 1.5 & 91 \\
\hline $6 e$ & $4-\mathrm{ClC}_{6} \mathrm{H}_{4}$ & morphilin-1-yl & 2.5 & 88 \\
\hline $6 f$ & $4-\mathrm{ClC}_{6} \mathrm{H}_{4}$ & pyrrolidin-1-yl & 2.0 & 80 \\
\hline $6 \mathrm{~g}$ & $4-\mathrm{BrC}_{6} \mathrm{H}_{4}$ & piperidin-1-yl & 2.0 & 81 \\
\hline $6 \mathrm{~h}$ & $4-\mathrm{BrC}_{6} \mathrm{H}_{4}$ & morphilin-1-yl & 2.0 & 80 \\
\hline $6 \mathbf{i}$ & $4-\mathrm{BrC}_{6} \mathrm{H}_{4}$ & pyrrolidin-1-yl & 3.0 & 80 \\
\hline $6 j$ & $4-\mathrm{O}_{2} \mathrm{NC}_{6} \mathrm{H}_{4}$ & piperidin-1-yl & 3.0 & 76 \\
\hline $6 k$ & $4-\mathrm{O}_{2} \mathrm{NC}_{6} \mathrm{H}_{4}$ & morphilin-1-yl & 4.0 & 71 \\
\hline 61 & $4-\mathrm{O}_{2} \mathrm{NC}_{6} \mathrm{H}_{4}$ & pyrrolidin-1-yl & 3.5 & 73 \\
\hline $6 \mathrm{~m}$ & 4-MeOC $6 \mathrm{H}_{4}$ & piperidin-1-yl & 1.5 & 89 \\
\hline $6 n$ & 4- $\mathrm{MeOC}_{6} \mathrm{H}_{4}$ & morphilin-1-yl & 2.5 & 81 \\
\hline 60 & 4- $\mathrm{MeOC}_{6} \mathrm{H}_{4}$ & pyrrolidin-1-yl & 2.0 & 83 \\
\hline $6 p$ & 4-pyridyl & piperidin-1-yl & 2.0 & 89 \\
\hline $6 q$ & 4-pyridyl & morphilin-1-yl & 3.0 & 79 \\
\hline $6 r$ & 4-pyridyl & pyrrolidin-1-yl & 2.0 & 82 \\
\hline
\end{tabular}

${ }^{a}$ Reaction conditions: 2-arylidine-1-thia-4-azaspiro[4.5]decan-3-ones (5a-f) $(10 \mathrm{mmol})$, formaldehyde $(15 \mathrm{mmol})$, and secondary amine $(15 \mathrm{mmol})$ in $10 \mathrm{~mL}$ absolute ethanol at room temperature.

${ }^{\mathrm{b}}$ Isolated yields.
2-(4-Methylbenzylidene)-1-thia-4-azaspiro[4.5]decan-3-one (5e))

Yellow crystals; mp 230-231 ${ }^{\circ} \mathrm{C}$; IR: $3200(\mathrm{NH}), 3090$ (CH arom.), 1695 (C= O), 690 (C-S); ${ }^{1} \mathrm{H}$ NMR (DMSO$\left.\mathrm{d}_{6}\right)$ : 1.10-2.05 (m, 10H, $\left.5 \times \mathrm{CH}_{2}\right), 3.85\left(\mathrm{~s}, 3 \mathrm{H}, \mathrm{CH}_{3}\right), 7.05$ (d, $2 \mathrm{H}, 2 \mathrm{CH}, J=7.3), 7.60$ (d, $2 \mathrm{H}, 2 \mathrm{CH}, J=7.3), 7.85$ (s, $1 \mathrm{H}, \mathrm{CH}), 8.90(\mathrm{~s}, 1 \mathrm{H}, \mathrm{NH}) \mathrm{ppm} ;{ }^{13} \mathrm{C} \mathrm{NMR}: 22.2\left(2 \mathrm{CH}_{2}\right)$, $24.7\left(\mathrm{CH}_{2}\right), 26.1\left(2 \mathrm{CH}_{2}\right), 42.1\left(\mathrm{CH}_{3}\right), 47.3$ (spiro C), 121.9 $(\mathrm{CH}), 125.3(2 \mathrm{CH}), 126.7(2 \mathrm{CH}), 129.2(\mathrm{C}), 131.5(\mathrm{C})$, 136.9 (C), $171.3(\mathrm{C}=\mathrm{O}) \mathrm{ppm}$; EI-MS, $\mathrm{m} / z(\%): 290.54$ (12) $\left[M^{+}+1\right], 289.02(40)\left[M^{+}\right], 274.01$ (17), 246.12 (100.0). Calc. for $\mathrm{C}_{16} \mathrm{H}_{19} \mathrm{NO}_{2} \mathrm{~S}: \mathrm{C}, 66.41 ; \mathrm{H}, 6.62 ; \mathrm{N}$, 4.84; S, 11.08. Found: C, 66.09; H, 6.45; N, 4.68; S, 10.85.

\section{2-(Pyridin-4-ylmethylene)-1-thia-4-azaspiro[4.5]decan-3- one (5f))}

Pale yellow crystals; mp $225-227^{\circ} \mathrm{C}$; IR: $3190(\mathrm{NH}), 3090$ (CH arom.), 1695 (C= O), 695 (C-S); ${ }^{1} \mathrm{H}$ NMR (DMSO$\left.\mathrm{d}_{6}\right)$ : $1.05-2.10\left(\mathrm{~m}, 10 \mathrm{H}, 5 \mathrm{CH}_{2}\right), 7.00(\mathrm{~d}, 2 \mathrm{H}, 2 \mathrm{CH}, J=$ 6.8), 7.70 (d, 2H, 2CH, J=6.8), 7.80 (s, $1 \mathrm{H}, \mathrm{CH}$ ), 8.10 (s, $1 \mathrm{H}, \mathrm{NH}) \mathrm{ppm}$; EI-MS, $m / z(\%): 260.15(25)[M+], 217.15$ (100.0). Calc. for $\mathrm{C}_{14} \mathrm{H}_{16} \mathrm{~N}_{2} \mathrm{OS}$ : C, 64.58; $\mathrm{H}, 6.19 ; \mathrm{N}$, 10.76; S, 12.32. Found: C, 64.36; H, 6.00; N, 10.79; S, 12.20 .

\section{General procedure for synthesis of Mannich Bases (6a-r))}

To a solution of 2-benzylidene-1-thia-4-azaspiro[4.5]decan-3-one (5a) (0.259 g, $1 \mathrm{mmol})$ in $5 \mathrm{~mL}$ of absolute ethanol was added a mixture of sec. amine $(1.5 \mathrm{mmol})$ and aqueous formaldehyde $35 \%(0.2 \mathrm{~mL}, 1.5 \mathrm{mmol})$ also dissolved in $5 \mathrm{~mL}$ absolute ethanol. The reaction mixture was stirred for the desired time as monitored by TLC (Table 4), refrigerated for $24 \mathrm{~h}$ to form crystals. The crystalline product was separated by filtration, vacuum dried and recrystallized from ethanol.

\section{2-Benzylidene-4-(piperidin-1-ylmethyl)-1-thia-4- azaspiro[4.5]decan-3-one (6a))}

Pale yellow crystals; mp $119-120^{\circ} \mathrm{C}$; IR: $3030(\mathrm{CH}$ arom.), 2950 (CH aliph.), 1700 (C=O), 695 (C-S); ${ }^{1} \mathrm{H}$ NMR $\left(\mathrm{CDCl}_{3}\right): 1.38-1.51\left(\mathrm{~m}, 12 \mathrm{H}, 6 \mathrm{CH}_{2}\right), 2.10-2.37(\mathrm{~m}$, $\left.4 \mathrm{H}, 2 \mathrm{CH}_{2}\right), 2.43-2.48\left(\mathrm{~m}, 4 \mathrm{H}, 2 \mathrm{CH}_{2}\right), 4.08\left(\mathrm{~s}, 2 \mathrm{H}, \mathrm{CH}_{2}\right)$, 
Table 5 Bactericidal activity of Mannich bases (6a-r) using Ciprofloxacin as standard antibacterial reference ${ }^{a}$

\begin{tabular}{|c|c|c|c|}
\hline Compound & Staphylococcus aureus & Escherichia Coli & Pseudomonas aeroginosa \\
\hline $6 a$ & - & + & - \\
\hline $6 b$ & ++ & + & ++ \\
\hline $6 c$ & - & - & - \\
\hline $6 d$ & ++ & + & + \\
\hline $6 e$ & +++ & +++ & +++ \\
\hline $6 f$ & - & + & - \\
\hline $6 \mathrm{~g}$ & + & ++ & - \\
\hline $6 h$ & ++ & ++ & ++++ \\
\hline $6 i$ & - & - & + \\
\hline $6 \mathbf{j}$ & ++ & - & - \\
\hline $6 k$ & + & +++ & ++ \\
\hline 61 & - & - & - \\
\hline $6 m$ & +++ & +++ & ++ \\
\hline $6 n$ & ++++ & ++++ & +++ \\
\hline 60 & + & + & - \\
\hline $6 p$ & ++++ & + & ++ \\
\hline $6 q$ & ++++ & +++ & +++ \\
\hline $6 r$ & - & ++ & + \\
\hline Ciprofloxacin & ++++ & ++++ & ++++ \\
\hline
\end{tabular}

${ }^{a}$ The activities are based on the diameter of zones of inhibition in $\mathrm{mm}$. $50 \mu \mathrm{L}$ of stock solution was applied in each hole of each paper disk. +: $<15 \mathrm{~mm} ;++$ : $15-24 \mathrm{~mm}$; +++: 25-34 mm; ++++: 35-44 mm.

Table 6 Fungicidal activity of Mannich bases (6a-r) using Nystatin as standard antifungal reference ${ }^{a}$

\begin{tabular}{|c|c|c|c|}
\hline Compound & Aspergillus niger & Candida albicans & Fusarium oxysporium \\
\hline $6 a$ & + & + & + \\
\hline $6 b$ & + & - & + \\
\hline $6 c$ & - & - & - \\
\hline $6 d$ & +++ & +++ & +++ \\
\hline $6 e$ & ++ & +++ & + \\
\hline $6 f$ & + & ++ & - \\
\hline $6 \mathrm{~g}$ & ++ & ++ & ++ \\
\hline $6 \mathrm{~h}$ & + & + & - \\
\hline $6 i$ & - & ++ & - \\
\hline $6 j$ & ++ & - & + \\
\hline $6 k$ & ++ & +++ & ++ \\
\hline 61 & +++ & ++++ & ++++ \\
\hline $6 m$ & ++ & - & - \\
\hline $6 n$ & + & + & ++ \\
\hline 60 & +++ & ++ & - \\
\hline $6 p$ & ++++ & +++ & +++ \\
\hline $6 q$ & +++ & ++ & +++ \\
\hline $6 r$ & + & + & + \\
\hline Nystatin & ++++ & ++++ & ++++ \\
\hline
\end{tabular}

${ }^{\mathrm{a}}$ The activities are based on the diameter of zones of inhibition in $\mathrm{mm}$. $50 \mu \mathrm{L}$ of stock solution was applied in each hole of each paper disk. $+:<15 \mathrm{~mm} ;++: 15-24 \mathrm{~mm}$; +++ : 25-34 mm; ++++: 35-44 mm. 
7.32-7.60 (m, 5H, Ph-H), 7.75 (s, 1H, CH) ppm; EI-MS, $m / z$ (\%): 357.05 (9) $\left[\mathrm{M}^{+}+1\right], 356.01$ (46) $\left[\mathrm{M}^{+}\right], 258.12$ (25), 215.65 (100.0). Calc. for $\mathrm{C}_{21} \mathrm{H}_{28} \mathrm{~N}_{2} \mathrm{OS}$ : C, 70.75; $\mathrm{H}$, 7.92; N, 7.86; S, 8.99. Found: C, 70.52; H, 7.80; N, 7.66; S, 8.78.

\section{2-Benzylidene-4-(morpholinomethyl)-1-thia-4- azaspiro[4.5]decan-3-one (6b))}

Pale yellow crystals; mp $135-137^{\circ} \mathrm{C}$; IR: $3020(\mathrm{CH}$ arom.), 2955 (CH aliph.), $1700(\mathrm{C}=\mathrm{O}), 695(\mathrm{C}-\mathrm{S}) ;{ }^{1} \mathrm{H}$ NMR (DMSO-d $\left.\mathrm{d}_{6}\right): 1.42-1.51\left(\mathrm{~m}, 6 \mathrm{H}, 3 \mathrm{CH}_{2}\right), 2.15-2.40$ $\left(\mathrm{m}, 4 \mathrm{H}, 2 \mathrm{CH}_{2}\right), 2.47\left(\mathrm{t}, 4 \mathrm{H}, 2 \mathrm{CH}_{2}, J=6.9\right), 3.59(\mathrm{t}, 4 \mathrm{H}$, $\left.2 \mathrm{CH}_{2}, J=6.9\right), 4.15$ (s, 2H, $\mathrm{CH}_{2}$ ), 7.33-7.61 (m, 5H, Ph$\mathrm{H}), 7.71$ (s, 1H, CH) ppm; EI-MS, $m / z$ (\%): 359.01 (5) $\left[\mathrm{M}^{+}+1\right], 358.00(32)\left[M^{+}\right], 258.09$ (17), 216.05 (100.0). Calc. for $\mathrm{C}_{20} \mathrm{H}_{26} \mathrm{~N}_{2} \mathrm{O}_{2} \mathrm{~S}: \mathrm{C}, 67.01 ; \mathrm{H}, 7.31 ; \mathrm{N}, 7.81 ; \mathrm{S}$, 8.94. Found: C, 66.80; H, 7.05; N, 7.65; S, 8.70.

\section{2-Benzylidene-4-(pyrrolidin-1-ylmethyl)-1-thia-4- azaspiro[4.5]decan-3-one (6c))}

Yellow crystals; mp $126-127^{\circ} \mathrm{C}$; IR: 3020 ( $\mathrm{CH}$ arom.), 2880 (CH aliph.), $1705(\mathrm{C}=\mathrm{O}), 695(\mathrm{C}-\mathrm{S}) ;{ }^{1} \mathrm{H}$ NMR $\left(\mathrm{CDCl}_{3}\right): 1.43-1.52\left(\mathrm{~m}, 6 \mathrm{H}, 3 \mathrm{CH}_{2}\right), 1.62\left(\mathrm{t}, 4 \mathrm{H}, 2 \mathrm{CH}_{2}, J=\right.$ 7.1), 2.17-2.40 (m, $\left.4 \mathrm{H}, 2 \mathrm{CH}_{2}\right), 2.53\left(\mathrm{t}, 4 \mathrm{H}, 2 \mathrm{CH}_{2}, J=7.1\right)$, 4.01 (s, 2H, $\left.\mathrm{CH}_{2}\right), 7.30-7.58(\mathrm{~m}, 5 \mathrm{H}, \mathrm{Ph}-\mathrm{H}), 7.60(\mathrm{~s}, 1 \mathrm{H}$, CH) ppm; EI-MS, $m / z$ (\%): 341.82 (15) $\left[M^{+}\right], 258.09$ (25), 215.85 (100.0). Calc. for $\mathrm{C}_{20} \mathrm{H}_{26} \mathrm{~N}_{2} \mathrm{OS}$ : C, 70.14; $\mathrm{H}$, 7.65; N, 8.18; S, 9.36. Found: C, 69.80; H, 7.43; N, 7.76; S, 9.08 .

\section{2-(4-Chlorobenzylidene)-4-(piperidin-1-ylmethyl)-1-thia-4- azaspiro[4.5]decan-3-one (6d))}

Yellow crystals; mp 120-122 $2^{\circ}$; IR: 3050 (CH arom.), 2955 (CH aliph.), 1700 (C=O), 695 (C-S); ${ }^{1} \mathrm{H}$ NMR $\left(\mathrm{CDCl}_{3}\right): 1.44-1.61\left(\mathrm{~m}, 12 \mathrm{H}, 6 \mathrm{CH}_{2}\right), 2.10-2.46(\mathrm{~m}, 8 \mathrm{H}$, $\left.4 \mathrm{CH}_{2}\right), 4.25\left(\mathrm{~s}, 2 \mathrm{H}, \mathrm{CH}_{2}\right), 7.40(\mathrm{~d}, 2 \mathrm{H}, 2 \mathrm{CH}, J=7.6), 7.60$ (s, $1 \mathrm{H}, \mathrm{CH}), 7.72(\mathrm{~d}, 2 \mathrm{H}, 2 \mathrm{CH}, J=7.6) \mathrm{ppm}$; EI-MS, $m / z$ (\%): 390.65 (32) $\left[M^{+}\right], 292.12$ (16), 215.05 (100.0). Calc. for $\mathrm{C}_{21} \mathrm{H}_{27} \mathrm{ClN}_{2} \mathrm{OS}$ : C, 64.51; $\mathrm{H}, 6.96 ; \mathrm{Cl}, 9.07 ; \mathrm{N}, 7.17 ; \mathrm{S}$, 8.20. Found: C, 64.22; H, 6.66; Cl, 8.81; N, 7.00; S, 7.92.

\section{2-(4-Chlorobenzylidene)-4-(morpholinomethyl)-1-thia-4- azaspiro[4.5]decan-3-one (6e))}

Pale yellow crystals; mp $141-143^{\circ} \mathrm{C}$; IR: $3050(\mathrm{CH}$ arom.), 2880 (CH aliph.), 1700 (C=O), $695(\mathrm{C}-\mathrm{S}) ;{ }^{1} \mathrm{H}$ NMR (DMSO-d $\left.\mathrm{d}_{6}\right): 1.44-1.56\left(\mathrm{~m}, 6 \mathrm{H}, 3 \mathrm{CH}_{2}\right), 2.18-2.45$ $\left(\mathrm{m}, 4 \mathrm{H}, 2 \mathrm{CH}_{2}\right), 2.52\left(\mathrm{t}, 4 \mathrm{H}, 2 \mathrm{CH}_{2}, J=7.8\right), 3.50(\mathrm{t}, 4 \mathrm{H}$, $\left.2 \mathrm{CH}_{2}, J=7.9\right), 4.23\left(\mathrm{~s}, 2 \mathrm{H}, \mathrm{CH}_{2}\right), 7.38(\mathrm{~d}, 2 \mathrm{H}, 2 \mathrm{CH}, J=$ 6.5), $7.64(\mathrm{~s}, 1 \mathrm{H}, \mathrm{CH}), 7.70(\mathrm{~d}, 2 \mathrm{H}, 2 \mathrm{CH}, J=6.5) \mathrm{ppm}$; EI-MS, $m / z$ (\%): 392.71 (56) $\left[M^{+}\right], 292.00$ (20), 216.65 (100.0). Calc. for $\mathrm{C}_{20} \mathrm{H}_{25} \mathrm{ClN}_{2} \mathrm{O}_{2} \mathrm{~S}: \mathrm{C}, 61.13 ; \mathrm{H}, 6.41 ; \mathrm{Cl}$,
9.02; N, 7.13; S, 8.16. Found: C, 60.86; H, 6.20; Cl, 8.80; N, 6.90; S, 7.88.

2-(4-Chlorobenzylidene)-4-(pyrrolidin-1-ylmethyl)-1-thia-4azaspiro[4.5]decan-3-one (6f))

Pale yellow crystals; mp $133-135^{\circ} \mathrm{C}$; IR: $3035(\mathrm{CH}$ arom.), 2895 (CH aliph.), $1700(\mathrm{C}=\mathrm{O}), 690(\mathrm{C}-\mathrm{S}) ;{ }^{1} \mathrm{H}$ NMR $\left(\mathrm{CDCl}_{3}\right): 1.40-1.51\left(\mathrm{~m}, 6 \mathrm{H}, 3 \mathrm{CH}_{2}\right), 1.64(\mathrm{t}, 4 \mathrm{H}$, $\left.2 \mathrm{CH}_{2}, J=7.5\right), 2.25-2.38\left(\mathrm{~m}, 4 \mathrm{H}, 2 \mathrm{CH}_{2}\right), 2.62(\mathrm{t}, 4 \mathrm{H}$, $\left.2 \mathrm{CH}_{2}, J=7.5\right), 4.12\left(\mathrm{~s}, 2 \mathrm{H}, \mathrm{CH}_{2}\right), 7.35(\mathrm{~d}, 2 \mathrm{H}, 2 \mathrm{CH}, J=$ 6.3), $7.62(\mathrm{~d}, 2 \mathrm{H}, 2 \mathrm{CH}, J=6.3), 7.64(\mathrm{~s}, 1 \mathrm{H}, \mathrm{CH}) \mathrm{ppm}$; EI-MS, $m / z$ (\%): 375.90 (18) [ $\left[M^{+}\right], 291.89$ (30), 215.70 (100.0). Calc. for $\mathrm{C}_{20} \mathrm{H}_{25} \mathrm{ClN}_{2} \mathrm{OS}$ : C, 63.73; $\mathrm{H}, 6.68 ; \mathrm{Cl}$, 9.41; N, 7.43; S, 8.51. Found: C, 63.45; H, 6.70; Cl, 9.19; N, 7.20; S, 8.27.

2-(4-Bromobenzylidene)-4-(piperidin-1-ylmethyl)-1-thia-4azaspiro[4.5]decan-3-one (6g))

Pale yellow crystals; mp $133-135^{\circ} \mathrm{C}$; IR: $3050(\mathrm{CH}$ arom.), 2940 ( $\mathrm{CH}$ aliph.), 1705 (C= O), 690 (C-S); ${ }^{1} \mathrm{H}$ $\operatorname{NMR}\left(\mathrm{CDCl}_{3}\right): 1.38-1.50\left(\mathrm{~m}, 12 \mathrm{H}, 6 \mathrm{CH}_{2}\right), 2.05-2.47(\mathrm{~m}$, $8 \mathrm{H}, 4 \mathrm{CH}_{2}$ ), $4.05\left(\mathrm{~s}, 2 \mathrm{H}, \mathrm{CH}_{2}\right), 7.31$ (d, $2 \mathrm{H}, 2 \mathrm{CH}, J=7.1$ ), 7.58 (s, 1H, CH), 7.62 (d, 2H, 2CH, J= 7.1) ppm; EI-MS, $m / z$ (\%): 436.15 (19) $[\mathrm{M}+2], 434.09$ (21) $\left[\mathrm{M}^{+}\right], 335.60$ (11), 216.95 (100.0). Calc. for $\mathrm{C}_{21} \mathrm{H}_{27} \mathrm{BrN}_{2} \mathrm{OS}$ : C, 57.93; $\mathrm{H}, 6.25 ; \mathrm{Br}, 18.35 ; \mathrm{N}, 6.43 ; \mathrm{S}, 7.36$. Found: C, 57.68; H, 6.00; Br, 18.05; N, 6.22; S, 7.10.

\section{2-(4-Bromobenzylidene)-4-(morpholinomethyl)-1-thia-4- azaspiro[4.5]decan-3-one (6h))}

Pale yellow crystals; mp $152-153^{\circ} \mathrm{C}$; IR: $3030(\mathrm{CH}$ arom.), 2850 (CH aliph.), 1705 (C= O), 695 (C-S); ${ }^{1} \mathrm{H}$ NMR (DMSO-d $\left.\mathrm{d}_{6}\right): 1.40-1.53\left(\mathrm{~m}, 6 \mathrm{H}, 3 \mathrm{CH}_{2}\right), 2.10-2.40$ $\left(\mathrm{m}, 4 \mathrm{H}, 2 \mathrm{CH}_{2}\right), 2.52\left(\mathrm{t}, 4 \mathrm{H}, 2 \mathrm{CH}_{2}, J=6.9\right), 3.42(\mathrm{t}, 4 \mathrm{H}$, $\left.2 \mathrm{CH}_{2}, J=6.9\right), 4.09\left(\mathrm{~s}, 2 \mathrm{H}, \mathrm{CH}_{2}\right), 7.35(\mathrm{~d}, 2 \mathrm{H}, 2 \mathrm{CH}, J=$ 6.5), 7.65 (s, $1 \mathrm{H}, \mathrm{CH}), 7.58(\mathrm{~d}, 2 \mathrm{H}, 2 \mathrm{CH}, J=6.5) \mathrm{ppm}$; EI-MS, $m / z$ (\%): 438.01 (9) $\left[M^{+}+2\right], 436.2(10)[M+]$, 336.20 (43), 216.75 (100.0). Calc. for $\mathrm{C}_{20} \mathrm{H}_{25} \mathrm{BrN}_{2} \mathrm{O}_{2} \mathrm{~S}$ : C, 54.92; H, 5.76; Br, 18.27; N, 6.40; S, 7.33 Found: C, 54.75; H, 5.80; Br, 18.05; N, 6.17; S, 7.09.

2-(4-Bromobenzylidene)-4-(pyrrolidin-1-ylmethyl)-1-thia-4azaspiro[4.5]decan-3-one (6i))

Pale yellow crystals; mp $142-143^{\circ} \mathrm{C}$; IR: $3040(\mathrm{CH}$ arom.), 2890 (CH aliph.), 1700 (C=O), $695(\mathrm{C}-\mathrm{S}) ;{ }^{1} \mathrm{H}$ NMR $\left(\mathrm{CDCl}_{3}\right): 1.41-1.50\left(\mathrm{~m}, 6 \mathrm{H}, 3 \mathrm{CH}_{2}\right), 1.60(\mathrm{t}, 4 \mathrm{H}$, $\left.2 \mathrm{CH}_{2}, J=7.1\right), 2.20-2.35\left(\mathrm{~m}, 4 \mathrm{H}, 2 \mathrm{CH}_{2}\right), 2.59(\mathrm{t}, 4 \mathrm{H}$, $\left.2 \mathrm{CH}_{2}, J=7.1\right), 4.10\left(\mathrm{~s}, 2 \mathrm{H}, \mathrm{CH}_{2}\right), 7.33$ (d, $2 \mathrm{H}, 2 \mathrm{CH}, J=$ 6.5), 7.47 (s, 1H, CH), 7.55 (d, 2H, 2CH, J=6.5) ppm; EI-MS, $m / z$ (\%): 420.96 (26) $\left[M^{+}\right], 335.89$ (45), 216.71 (100.0). Calc. for $\mathrm{C}_{20} \mathrm{H}_{25} \mathrm{BrN}_{2} \mathrm{OS}$ : C, 57.00; H, 5.98; $\mathrm{Br}$, 18.96; N, 6.65; S, 7.61. Found: C, 56.15; H, 5.69; Br, 18.80; N, 6.50; S, 7.57. 
2-(4-Nitrobenzylidene)-4-(piperidin-1-ylmethyl)-1-thia-4azaspiro[4.5]decan-3-one (6j))

Yellow crystals; mp $142-144^{\circ} \mathrm{C}$; IR: 3050 (CH arom.), 2900 (CH aliph.), 1700 (C=O), 690 (C-S); ${ }^{1} \mathrm{H}$ NMR $\left(\mathrm{CDCl}_{3}\right)$ : 1.45-1.61 (m, 12H, 6CH $), 2.17-2.49(\mathrm{~m}, 8 \mathrm{H}$, $\left.4 \mathrm{CH}_{2}\right), 4.26\left(\mathrm{~s}, 2 \mathrm{H}, \mathrm{CH}_{2}\right), 7.92(\mathrm{~s}, 1 \mathrm{H}, \mathrm{CH}), 8.14(\mathrm{~d}, 2 \mathrm{H}$, $2 \mathrm{CH}, J=6.8), 8.35$ (d, $2 \mathrm{H}, 2 \mathrm{CH}, J=6.8) \mathrm{ppm}$; EI-MS, $m / z$ (\%): 402.16 (15) $\left[M^{+}+1\right], 303.62$ (22), 215.85 (100.0). Calc. for $\mathrm{C}_{21} \mathrm{H}_{27} \mathrm{~N}_{3} \mathrm{O}_{3} \mathrm{~S}$ : C, 62.82; $\mathrm{H}, 6.78 ; \mathrm{N}, 10.47 ; \mathrm{S}$, 7.99. Found: C, 62.60; H, 6.57; N, 10.49; S, 7.76.

\section{4-(Morpholinomethyl)-2-(4-nitrobenzylidene)-1-thia-4- azaspiro[4.5]decan-3-one (6k))}

Yellow crystals; mp $158-160^{\circ} \mathrm{C}$; IR: 3020 (CH arom.), 2900 (CH aliph.), 1700 (C=O), 695 (C-S); ${ }^{1} \mathrm{H}$ NMR (DMSO-d $)_{6}$ : 1.45-1.58 (m, 6H, 3CH $\left.\mathrm{CH}_{2}\right), 2.20-2.52(\mathrm{~m}, 4 \mathrm{H}$, $\left.2 \mathrm{CH}_{2}\right), 2.55\left(\mathrm{t}, 4 \mathrm{H}, 2 \mathrm{CH}_{2}, J=7.5\right), 3.54\left(\mathrm{t}, 4 \mathrm{H}, 2 \mathrm{CH}_{2}, J=\right.$ 7.5), $4.20\left(\mathrm{~s}, 2 \mathrm{H}, \mathrm{CH}_{2}\right), 7.71(\mathrm{~s}, 1 \mathrm{H}, \mathrm{CH}), 8.08(\mathrm{~d}, 2 \mathrm{H}$, $2 \mathrm{CH}, J=6.8), 8.29$ (d, $2 \mathrm{H}, 2 \mathrm{CH}, J=6.8) \mathrm{ppm}$; EI-MS, $m / z$ (\%): 403.12 (41) $\left[M^{+}\right], 303.25$ (35), 215.36 (100.0). Calc. for $\mathrm{C}_{20} \mathrm{H}_{25} \mathrm{~N}_{3} \mathrm{O}_{4} \mathrm{~S}$ : C, 59.53; $\mathrm{H}, 6.25 ; \mathrm{N}, 10.41 ; \mathrm{S}, 7.95$. Found: C, 59.58; H, 6.01; N, 10.26; S, 7.71.

\section{2-(4-Nitrobenzylidene)-4-(pyrrolidin-1-ylmethyl)-1-thia-4- azaspiro[4.5]decan-3-one (6I))}

Yellow crystals; mp 149-151 ${ }^{\circ} \mathrm{C}$; IR: 3025 ( $\mathrm{CH}$ arom.), 2990 (CH aliph.), 1705 (C=O), 690 (C-S); ${ }^{1} \mathrm{H}$ NMR $\left(\mathrm{CDCl}_{3}\right): 1.43-1.52\left(\mathrm{~m}, 6 \mathrm{H}, 3 \mathrm{CH}_{2}\right), 1.61\left(\mathrm{t}, 4 \mathrm{H}, 2 \mathrm{CH}_{2}, J=\right.$ 6.9), 2.25-2.37 (m, $\left.4 \mathrm{H}, 2 \mathrm{CH}_{2}\right), 2.58\left(\mathrm{t}, 4 \mathrm{H}, 2 \mathrm{CH}_{2}, J=6.9\right)$, 4.20 (s, 2H, $\mathrm{CH}_{2}$ ), 7.41 (s, 1H, CH), 7.93 (d, 2H, 2CH, J = 6.8), $8.21(\mathrm{~d}, 2 \mathrm{H}, 2 \mathrm{CH}, J=6.8) \mathrm{ppm}$; EI-MS, $m / z(\%)$ : 387.20 (19) $\left[M^{+}\right], 303.10$ (44), 215.87 (100.0). Calc. for $\mathrm{C}_{20} \mathrm{H}_{25} \mathrm{~N}_{3} \mathrm{O}_{3} \mathrm{~S}: \mathrm{C}, 61.99 ; \mathrm{H}, 6.50 ; \mathrm{N}, 10.84 ; \mathrm{S}, 8.27$. Found: C, 62.05; H, 6.24; N, 10.70; S, 8.00.

2-(4-Methoxybenzylidene)-4-(piperidin-1-ylmethyl)-1-thia4-azaspiro[4.5]decan-3-one $(6 \mathrm{~m})$ )

Pale yellow crystals; mp $121-123^{\circ} \mathrm{C}$; IR: $3200(\mathrm{NH}), 3090$ (CH arom.), $1700(\mathrm{C}=\mathrm{O}), 690(\mathrm{C}-\mathrm{S}) ;{ }^{1} \mathrm{H}$ NMR $\left(\mathrm{CDCl}_{3}\right)$ : 1.42-1.53 (m, $\left.12 \mathrm{H}, 6 \mathrm{CH}_{2}\right), 2.15-2.41\left(\mathrm{~m}, 8 \mathrm{H}, 4 \mathrm{CH}_{2}\right)$, 3.50 (s, 3H, $\left.\mathrm{CH}_{3}\right), 4.10\left(\mathrm{~s}, 2 \mathrm{H}, \mathrm{CH}_{2}\right), 6.92$ (d, 2H, 2CH, J = 6.8), 7.45 (d, 2H, 2CH, J=6.8), $7.56(\mathrm{~s}, 1 \mathrm{H}, \mathrm{CH}) \mathrm{ppm}$; EI-MS, $m / z$ (\%): 386.20 (15) $\left[\mathrm{M}^{+}\right], 288$ (45), 216.05 (100.0). Calc. for $\mathrm{C}_{22} \mathrm{H}_{30} \mathrm{~N}_{2} \mathrm{O}_{2} \mathrm{~S}: \mathrm{C}, 68.36 ; \mathrm{H}, 7.82 ; \mathrm{N}$, 7.25; S, 8.30. Found: C, 68.39; H, 7.70; N, 7.00; S, 8.02.

2-(4-Methoxybenzylidene)-4-(morpholinomethyl)-1-thia-4azaspiro[4.5]decan-3-one (6n))

Pale yellow crystals; mp $133-135^{\circ} \mathrm{C}$; IR: $3050(\mathrm{CH}$ arom.), 2950 (CH aliph.), 1700 (C=O), 695 (C-S); ${ }^{1} \mathrm{H}$ NMR $\left(\mathrm{CDCl}_{3}\right): 1.41-1.52\left(\mathrm{~m}, 6 \mathrm{H}, 3 \mathrm{CH}_{2}\right), 2.15-2.46(\mathrm{~m}$, $\left.4 \mathrm{H}, 2 \mathrm{CH}_{2}\right), 2.51\left(\mathrm{t}, 4 \mathrm{H}, 2 \mathrm{CH}_{2}, J=7.1\right), 3.50\left(\mathrm{t}, 4 \mathrm{H}, 2 \mathrm{CH}_{2}\right.$, $J=7.1$ ), 4.08 (s, 2H, $\mathrm{CH}_{2}$ ), 7.01 (d, $\left.2 \mathrm{H}, 2 \mathrm{CH}, J=6.9\right), 7.60$ (d, 2H, 2CH, J=6.9), 7.69 (s, 1H, CH) ppm; EI-MS, $m / z$
(\%): 388.19 (20) $\left[M^{+}\right], 288.75$ (51), 215.06 (100.0). Calc. for $\mathrm{C}_{21} \mathrm{H}_{28} \mathrm{~N}_{2} \mathrm{O}_{3} \mathrm{~S}$ : C, 64.92; $\mathrm{H}, 7.26 ; \mathrm{N}, 7.21 ; \mathrm{S}, 8.25$. Found: C, 64.70; H, 7.00; N, 7.30; S, 8.01.

2-(4-Methoxybenzylidene)-4-(pyrrolidin-1-ylmethyl)-1-thia4-azaspiro[4.5]decan-3-one (6o))

Yellow crystals; mp 140-142 ${ }^{\circ} \mathrm{C}$; IR: 3050 ( $\mathrm{CH}$ arom.), 2900 (CH aliph.), 1700 (C=O), 695 (C-S); ${ }^{1} \mathrm{H}$ NMR $\left(\mathrm{CDCl}_{3}\right): 1.40-1.49\left(\mathrm{~m}, 6 \mathrm{H}, 3 \mathrm{CH}_{2}\right), 1.60\left(\mathrm{t}, 4 \mathrm{H}, 2 \mathrm{CH}_{2}\right)$, 2.20-2.32 (m, $4 \mathrm{H}, 2 \mathrm{CH}_{2}$ ), $2.55\left(\mathrm{t}, 4 \mathrm{H}, 2 \mathrm{CH}_{2}, J=7.3\right), 4.15$ (s, $\left.2 \mathrm{H}, \mathrm{CH}_{2}, J=7.3\right), 7.01$ (d, $\left.2 \mathrm{H}, 2 \mathrm{CH}, J=6.8\right), 7.26$ (d, $2 \mathrm{H}, 2 \mathrm{CH}, J=6.8), 7.29$ (s, $1 \mathrm{H}, \mathrm{CH}) \mathrm{ppm}$; EI-MS, $m / z(\%)$ : 372.19 (32) $\left[M^{+}\right], 289.02$ (35), 215.97 (100.0). Calc. for $\mathrm{C}_{21} \mathrm{H}_{28} \mathrm{~N}_{2} \mathrm{O}_{2} \mathrm{~S}: \mathrm{C}, 67.71 ; \mathrm{H}, 7.58 ; \mathrm{N}, 7.52 ; \mathrm{S}, 8.61$. Found: C, 67.58; H, 7.60; N, 7.40; S, 8.45.

4-(Piperidin-1-ylmethyl)-2-(pyridin-4-ylmethylene)-1-thia4-azaspiro[4.5]decan-3-one (6p))

Pale yellow crystals; mp $111-113^{\circ} \mathrm{C}$; IR: $3050(\mathrm{CH}$ arom.), 1695 (C=O), 690 (C-S); ${ }^{1} \mathrm{H}$ NMR $\left(\mathrm{CDCl}_{3}\right)$ : 1.40-1.56 (m, 12H, 6CH $\mathrm{CH}_{2}, 2.01-2.55\left(\mathrm{~m}, 8 \mathrm{H}, 4 \mathrm{CH}_{2}\right)$, 4.00 (s, 2H, $\mathrm{CH}_{2}$ ), 7.05 (d, $\left.2 \mathrm{H}, 2 \mathrm{CH}, J=6.9\right), 7.30$ (s, $1 \mathrm{H}$, $\mathrm{CH}), 7.83$ (d, 2H, 2CH, $J=6.9$ ), ppm; EI-MS, $m / z(\%)$ : 357.15 (16) $[M+], 259.30$ (40), 217.15 (100.0). Calc. for $\mathrm{C}_{20} \mathrm{H}_{27} \mathrm{~N}_{3} \mathrm{OS}$ : C, 67.19; H, 7.61; N, 11.75; S, 8.97. Found: C, 67.25; H, 7.50; N, 11.80; S, 8.66.

4-(Morpholinomethyl)-2-(pyridin-4-ylmethylene)-1-thia-4azaspiro[4.5]decan-3-one (6q))

Pale yellow crystals; mp $125-127^{\circ} \mathrm{C}$; IR: $3050(\mathrm{CH}$ arom.), 1700 (C=O), 695 (C-S); ${ }^{1} \mathrm{H}$ NMR $\left(\mathrm{CDCl}_{3}\right)$ : 1.39-1.50 (m, 6H, 3CH $\left.\mathrm{CH}_{2}\right), 2.15-2.45\left(\mathrm{~m}, 4 \mathrm{H}, 2 \mathrm{CH}_{2}\right), 2.52$ $\left(\mathrm{t}, 4 \mathrm{H}, 2 \mathrm{CH}_{2}, J=7.1\right), 3.47\left(\mathrm{t}, 4 \mathrm{H}, 2 \mathrm{CH}_{2}, J=7.1\right), 4.10(\mathrm{~s}$, $\left.2 \mathrm{H}, \mathrm{CH}_{2}\right), 6.91(\mathrm{~d}, 2 \mathrm{H}, 2 \mathrm{CH}, J=6.9), 7.15(\mathrm{~s}, 1 \mathrm{H}, \mathrm{CH})$, 7.80 (d, 2H, 2CH, $J=6.9) \mathrm{ppm}$; EI-MS, $m / z(\%): 359.60$ (21) $[M+], 259.95$ (61), 216.95 (100.0). Calc. for $\mathrm{C}_{19} \mathrm{H}_{25} \mathrm{~N}_{3} \mathrm{O}_{2} \mathrm{~S}: \mathrm{C}, 63.48 ; \mathrm{H}, 7.01 ; \mathrm{N}, 11.69 ; \mathrm{S}, 8.92$. Found: C, 63.20; H, 6.85; N, 11.71; S, 8.70.

2-(Pyridin-4-ylmethylene)-4-(pyrrolidin-1-ylmethyl)-1-thia4-azaspiro[4.5]decan-3-one (6r))

Pale yellow crystals; mp $119-120^{\circ} \mathrm{C}$; IR: $3050(\mathrm{CH}$ arom.), 1695 (C=O), 690 (C-S); ${ }^{1} \mathrm{H}$ NMR $\left(\mathrm{CDCl}_{3}\right)$ : 1.40$1.49\left(\mathrm{~m}, 6 \mathrm{H}, 3 \mathrm{CH}_{2}\right), 1.59\left(\mathrm{t}, 4 \mathrm{H}, 2 \mathrm{CH}_{2}, J=7.2\right), 2.22-2.40$ $\left(\mathrm{m}, 4 \mathrm{H}, 2 \mathrm{CH}_{2}\right), 2.55\left(\mathrm{t}, 4 \mathrm{H}, 2 \mathrm{CH}_{2}, J=7.2\right), 4.08(\mathrm{~s}, 2 \mathrm{H}$, $\left.\mathrm{CH}_{2}\right), 6.98(\mathrm{~d}, 2 \mathrm{H}, 2 \mathrm{CH}, J=6.4), 7.20(\mathrm{~s}, 1 \mathrm{H}, \mathrm{CH}), 7.90$ (d, 2H, 2CH, $J=6.4$ ) ppm; EI-MS, $m / z(\%): 343.05$ (20) $[M+], 259.02$ (65), 216.65 (100.0). Calc. for $\mathrm{C}_{19} \mathrm{H}_{25} \mathrm{~N}_{3} \mathrm{OS}$ : C, 66.44; H, 7.34; N, 12.23; S, 9.34. Found: C, 66.50; H, 7.36; N, 12.00; S, 9.16 . 


\section{Antimicrobial screening Antibacterial activity}

The newly synthesized compounds were screened for their antibacterial activity against bacterial isolate namely Staphylococcus aureus (ATCC 29213) as Gram positive bacteria, Escherichia Coli (ATCC 25922), Pseudomonas aeroginosa (ATCC 27953) as Gram negative bacteria by cup-plate method [23]. The sterilized nutrient agar medium was distributed $100 \mathrm{ml}$ each in two $250 \mathrm{ml}$ conical flasks and allowed to cool to room temperature. To these media, 18-24 h grown bacterial subcultures were added and shaken thoroughly to ensure uniform distribution of organism throughout the medium. Then, this agar medium was distributed in equal portions, in sterilized Petri dishes, ensuring that each Petri dish contains about $20 \mathrm{ml}$ of the medium. The medium was then allowed for solidification. Then, cups were made with the help of a sterile cork borer (6 mm diameter) punching into the set of agar media.

The solutions of required concentration $(50 \mu \mathrm{g} / \mathrm{mL})$ of test compounds were prepared by dissolving the compounds in DMSO were filled into the cups with $1 \mathrm{~mL}$ of respective solution. Then, the Petri dishes were kept for incubation in an inverted position for $24-48 \mathrm{~h}$ at $37^{\circ} \mathrm{C}$ in an incubator. When growth inhibition zones were developed surrounding each cup, their diameter in $\mathrm{cm}$ was measured and compared with that of the Ciprofloxacin.

\section{Antifungal activity}

The newly synthesized compounds were screened for their antifungal activity against three fungal strains; Aspergillus niger, Candida albicans, Fusarium oxysporium at the concentration levels of $50 \mu \mathrm{g} / \mathrm{mL}$ by cup-plate method, using Nystatin as the standard. To the sterilized potato dextrose agar medium incubated for $72 \mathrm{~h}$, subculture of fungus were added and shaken thoroughly to ensure uniform distribution. Then, this was poured into previously sterilized and labeled Petri dishes and allowed to solidify. Then, with the help of a borer four cups were made in each plate. Two cups were filled with $0.1 \mathrm{ml}$ of two test dilutions and the other two cups with respective concentrations of standard dilutions. Then, the plates were left as it is for $2-3 \mathrm{~h}$ for diffusion and then they were kept for incubation at $37^{\circ} \mathrm{C}$ for $24 \mathrm{~h}$. Then the diameter of the zones of growth inhibition was measured and compared with that of standard.

\section{Competing interests}

The authors declare that they have no competing interests.

\section{Authors' contributions}

$\mathrm{EMH}$ designed the research, performed the experimental work, and shared in writing the manuscript; GSM analyzed the data and shared in writing the manuscript; KSK revised the manuscript. All authors read and approved the final manuscript.

\begin{abstract}
Acknowledgments
Authors are thankful to Assiut University and the Faculty of Science (Assiut - Egypt)

for providing financial assistance to carry out this work.
\end{abstract}

Received: 18 December 2014 Accepted: 29 April 2015

Published online: 10 May 2015

\section{References}

1. Jain AK, Vaidya A, Ravichandran V, Kashaw SK, Agrawal RK. Recent developments and biological activities of thiazolidinone derivatives: A review. Bioorg Med Chem. 2012;20:3378-95.

2. Lewis JR. Miscellaneous alkaloids: Amaryllidaceae, Sceletium, muscarine, imidazole, oxazole, peptide and other miscellaneous alkaloids. Nat Prod Rep. 1999;16:389-416.

3. Clemence F, Marter OL, Delevalle F, Benzoni J, Jouanen A, Jouquey S, et al. 4-Hydroxy-3-quinolinecarboxamides with antiarthritic and analgesic activities. J Med Chem. 1988;31:1453-62.

4. Tsuji K, Ishikawa H. Synthesis and Anti-pseudomonal Activity of New 2-Isocephems with a Dihydroxypyridone Moiety at C-7. Biorg Med Chem Lett. 1944:4:1601-6.

5. Patt WC, Hamilton HW, Taylor MD, Ryan MJ, Taylor DG, Connolly CJC, et al. Structure-activity relationships of a series of 2-amino-4-thiazole-containing renin inhibitors. J Med Chem. 1992;35:2562-72.

6. Metzger JV. Comprehensive Heterocyclic Chemistry, vol. 6. New York: Pergamon Press; 1984. p. 328.

7. Sridhar SK, Saravanan M, Ramesh A. Synthesis and Antibacterial Screening of Hydrazones, Schiff and Mannich Bases of Isatin Derivatives. Eur J Med Chem. 2001;36:615-25.

8. Pandeya SN, Sriram D. Synthesis and Screening for Antibacterial Activity of Schiff's and Mannich Bases of Isatin and its Derivatives. Acta Pharm Turc. 1998;40:33-8.

9. Pandeya SN, Yogeeswari P, Sriram D, Nath G. Synthesis, Antibacterial and Antifungal Activities of N-Mannich Bases of 3-[N2-pyrimethaminylimino] isatin. Indian J Pharm Sci. 2002;64:209-12.

10. Chhajed SS, Padwal MS. Antimicrobial Evaluation of Some Novel Schiff and Mannich Bases of Isatin and its Derivatives with Quinolin. Int J ChemTech Res. 2010;2:209-13.

11. Hamley P, Mclnally T, Tinker A: PCT Int. Appl. WO 98 46, 611, (Cl. CO7D491 10), 1998, 22 Oct. 1998, SE Appl. 97/1, 396, 15 Apr. 1997; 32 PP.; Chem. Abstr. $1998,129,316237 \mathrm{f}$.

12. Trani A, Dallanoce C, Panzone G, Ripamonti F, Goldstein BP, Ciabatti R. Semisynthetic derivatives of purpuromycin as potential topical agents for vaginal infections. J Med Chem. 1997;40:967-71.

13. Jain SC, Sinha J, Bhagat S, Errington W, Olsen CE. A Facile Synthesis of Novel Spiro-[Indole-pyrazolinyl-thiazolidine]-2,4'-dione. Synth Commun. 2003:33:563-77.

14. Hussein EM. One-pot, Three-component, Green synthesis of Some Indeno[2',3':5,6] pyrido[2,1-b]benzothiazoles and Indeno[2',3'-e]thiazolo [3,2-a]pyridines. Heterocycl Lett. 2012;2:19-26.

15. Hussein EM. Enviro-economic, Ultrasound-assisted One-pot, Three-component Synthesis of Pyrido[2,3-d]pyrimidines in Aqueous Medium. Z Naturforsch. 2012;67b:231-7.

16. Hussein EM, El-Khawaga AM. Simple and Clean Procedure for Three-component Syntheses of Spiro\{pyrido[2,1-b]benzothiazole-3,3'-indolines\} and Spiro \{thiazolo[3,2-a]pyridine-7,3'-indolines\} in Aqueous Medium. J Heterocycl Chem. 2012;49:1296-301.

17. Hussein EM. Ultrasound-promoted Efficient Domino Reaction for One-pot synthesis of Spiro-5-cyanopyrimidines: A Rapid Procedure. Monatsh Chem. 2013;144:1691-7.

18. Hussein EM. Ammonium Chloride-Catalyzed Four-Component Sonochemical Synthesis of Novel Hexahydroquinolines Bearing a Sulfonamide Moiety. Russian J Org Chem. 2015;1:54-64.

19. Hussein EM, Abdel-Monem Ml. Regioselective Synthesis and Anti-inflammatory Activity of Novel Dispiro[pyrazolidine-4,3'-pyrrolidine-2',3"-indoline]-2",3,5-triones. ARKIVOC. 2011;10:85-98.

20. Hussein EM, Abdel-Monem MI. Regioselective Synthesis of Dispiro [indane-2,3'-pyrrolidine-2',3"-indoline]-1,2",3-triones and Evaluation of Their Anti-inflammatory Activities. Int Res J Pharm Pharmacol. 2012;2:45-51.

21. Abdel-Mohsen SA, Hussein EM. A Green Synthetic Approach to the Synthesis of Schiff Bases from 4-Amino-2-thioxo-1,3-diazaspiro[5.5]undec-4- 
ene-5-carbonitrile as Potential Anti-inflammatory. Agents Russian j Bioorg Chem. 2014;40:343-9.

22. Crossley NS. Carl Djerassi. Kielczewski MA: Studies in Organic Sulphur Compounds. Part XVII. The Synthesis of Previously Inaccessible Acylated Enamines by Desulphurisation of Thiazolidines. J. Chem. Soc; 1965. p. 6253-64.

23. Vogel HG. Drug discovery and evaluation, pharmacological assay. 2nd ed. New York: Springer; 2002. p. 670

\section{Publish with ChemistryCentral and every scientist can read your work free of charge \\ "Open access provides opportunities to our colleagues in other parts of the globe, by allowing anyone to view the content free of charge." \\ W. Jeffery Hurst, The Hershey Company. \\ - available free of charge to the entire scientific community \\ - peer reviewed and published immediately upon acceptance \\ - cited in PubMed and archived on PubMed Central \\ - yours - you keep the copyright \\ Submit your manuscript here: \\ http://www.chemistrycentral.com/manuscript/<smiles>c1ccccc1</smiles> 